\title{
Article \\ Clinical Spectrum of SCN5A Channelopathy in Children with Primary Electrical Disease and Structurally Normal Hearts
}

\author{
Teresa Villarreal-Molina ${ }^{1}{ }^{\mathbb{D}}$, Gabriela Paola García-Ordóñez ${ }^{1}$, Álvaro E. Reyes-Quintero ${ }^{2}{ }^{\circledR}$, \\ Mayra Domínguez-Pérez ${ }^{1}{ }^{\mathbb{D}}$, Leonor Jacobo-Albavera ${ }^{1} \mathbb{D}$, Santiago Nava ${ }^{2}$, Alessandra Carnevale ${ }^{3}$, \\ Argelia Medeiros-Domingo ${ }^{4, *}$ and Pedro Iturralde ${ }^{2, *}$ \\ 1 Laboratory of Cardiovascular Genomics, National Institute of Genomic Medicine, Mexico City 14610, Mexico; \\ mvillareal@inmegen.gob.mx (T.V.-M.); gabrielapgarciao12@gmail.com (G.P.G.-O.); \\ mdominguez@inmegen.gob.mx (M.D.-P.); ljacobo@inmegen.gob.mx (L.J.-A.) \\ 2 Departament of Electrocardiology, National Institute of Cardiology "Ignacio Chávez", \\ Mexico City 14080, Mexico; alvaroequintero@gmail.com (Á.E.R.-Q.); santiagonavat@hotmail.com (S.N.) \\ 3 Laboratory of Mendelian Diseases, National Institute of Genomic Medicine, Mexico City 14610, Mexico; \\ acarnevale@inmegen.gob.mx \\ 4 Swiss DNAlysis, Cardiogenetics, 8600 Dübendorf, Switzerland \\ * Correspondence: argelia.medeiros@hin.ch (A.M.-D.); pedroi@yahoo.com (P.I.); Tel.: +52-55-5107-3280 (P.I.)
}

Citation: Villarreal-Molina, T.; García-Ordóñez, G.P.; Reyes-Quintero,

Á.E.; Domínguez-Pérez, M.; Jacobo-Albavera, L.; Nava, S.; Carnevale, A.; Medeiros-Domingo, A.; Iturralde, P. Clinical Spectrum of SCN5A Channelopathy in Children with Primary Electrical Disease and Structurally Normal Hearts. Genes 2022, 13, 16. https://doi.org/ $10.3390 /$ genes 13010016

Academic Editor: Lars Allan Larsen

Received: 30 October 2021

Accepted: 18 December 2021

Published: 22 December 2021

Publisher's Note: MDPI stays neutral with regard to jurisdictional claims in published maps and institutional affiliations.

Copyright: (C) 2021 by the authors. Licensee MDPI, Basel, Switzerland. This article is an open access article distributed under the terms and conditions of the Creative Commons Attribution (CC BY) license (https:// creativecommons.org/licenses/by/ $4.0 /$ )

\begin{abstract}
Sodium voltage-gated channel $\alpha$ subunit 5 (SCN5A)-mutations may cause an array of arrhythmogenic syndromes most frequently as an autosomal dominant trait, with incomplete penetrance, variable expressivity and male predominance. In the present study, we retrospectively describe a group of Mexican patients with SCN5A-disease causing variants in whom the onset of symptoms occurred in the pediatric age range. The study included 17 patients with clinical diagnosis of primary electrical disease, at least one $S C N 5 A$ pathogenic or likely pathogenic mutation and age of onset $<18$ years, and all available first- and second-degree relatives. Fifteen patients ( $88.2 \%)$ were male, and sixteen independent variants were found (twelve missense, three truncating and one complex inframe deletion/insertion). The frequency of compound heterozygosity was remarkably high $(3 / 17,17.6 \%)$, with early childhood onset and severe disease. Overall, $70.6 \%$ of pediatric patients presented with overlap syndrome, $11.8 \%$ with isolated sick sinus syndrome, $11.8 \%$ with isolated Brugada syndrome (BrS) and 5.9\% with isolated type 3 long QT syndrome (LQTS). A total of 24/45 SCN5A mutation carriers were affected (overall penetrance $53.3 \%$ ), and penetrance was higher in males (63.3\%, 19 affected/30 mutation carriers) than in females (33.3\%, 5 affected/15 carriers). In conclusion, pediatric patients with $S C N A$-disease causing variants presented mainly as overlap syndrome, with predominant loss-of-function phenotypes of sick sinus syndrome (SSS), progressive cardiac conduction disease (PCCD) and ventricular arrhythmias.
\end{abstract}

Keywords: SCN5A-channelopathy; childhood onset; overlap syndrome; sick sinus syndrome; progressive cardiac conduction disease; Brugada syndrome; idiopathic ventricular tachycardia; long QT syndrome; compound heterozygosity

\section{Introduction}

The function of the cardiac voltage-gated sodium channel $\alpha$ subunit protein $\left(\mathrm{Na}_{\mathrm{V}} 1.5\right)$ is crucial because it initiates the cardiac action potential by generating the inward sodium current $\left(\mathrm{I}_{\mathrm{Na}}\right)$, which mediates cardiomyocyte excitability and impulse conduction in the myocardium and specialized conduction system. This channel also generates the late sodium current $\left(\mathrm{I}_{\mathrm{NaL}}\right)$, which plays a role in repolarization and refractoriness [1]. The $\alpha$ subunit of $\mathrm{Na}_{\mathrm{V}} 1.5$ is a $220 \mathrm{kDa}$ transmembrane protein encoded by the sodium voltage-gated channel $\alpha$ subunit (SCN5A) gene, consisting of a cytoplasmic N-terminal domain, four homologous transmembrane domains (TMD, DI-DIV) and a cytoplasmic C-terminal domain. Each TMD has six transmembrane $\alpha$-helix segments (S1-S6) connected by cytoplasmic linkers [2]. Although sodium channel $\alpha$-subunits are traditionally believed to form functional 
monomers, some SCN5A mutation studies in patients with inherited arrhythmias indicate that oligomerization of the sodium channel $\alpha$-subunits may occur, and recent experiments suggested that sodium channel $\alpha$-subunits physically interact, assemble, function and gate as a dimer [3].

SCN5A-mutations may cause several arrhythmogenic phenotypes most frequently inherited in an autosomal dominant fashion [4,5]. These mutations cause an array of arrhythmogenic syndromes including dilated, arrhythmogenic or non-compaction cardiomyopathy, or arrhythmogenic syndromes with minimal or no structural defects such as sick sinus syndrome (SSS), progressive cardiac conduction disease (PCCD), type 1 Brugada syndrome (BrS), familial atrial fibrillation, idiopathic ventricular tachycardia (VT), multifocal ectopic Purkinje-related premature contractions (MEPPC), and type 3 long QT syndrome (LQTS) [6]. Characteristically, SCN5A disease-causing mutations show incomplete penetrance, variable expressivity and male predominance for reasons that are not fully understood, which include both genetic background and environmental factors [7].

Early age of onset is associated with severity in many genetic diseases. Although there are many isolated case-reports of $S C N 5 A$-channelopathy in children in the medical literature [8-14], few studies have analyzed SCN5A-channelopathy cohorts where onset of symptoms occurs in pediatric patients. A large prospective multi-center pediatric cohort of SCN5A mutation-positive neonates reported that $67.9 \%$ were asymptomatic at diagnosis, while age $<1$ year at diagnosis, compound heterozygous mutations, and mutations with both gain- and loss-of-function were identified as independent risk factors for cardiac events [15]. In the present study, we retrospectively describe a group of Mexican patients with $S C N 5 A$-disease causing variants in whom the onset of symptoms occurred in the pediatric age range, attending the Ignacio Chávez National Institute of Cardiology over a 15 year period. This study provides insight, which can potentially be of aid for diagnosis, phenotypic characterization and to define therapeutic approaches in pediatric patients with SCN5A-disease causing variants.

\section{Materials and Methods}

\subsection{Subjects}

The study included a group of pediatric patients (age of onset $<18$ years) recruited from the Department of Cardiac Electrophysiology at the National Institute of Cardiology "Ignacio Chavez" in Mexico City over a 15-year period (2005 to 2020). All patients had a clinical diagnosis of primary electrical disease (SSS, PCCD, BrS, VT, LQTS) and a sodium channel disease-causing variant. All available first- and second-degree relatives were also included in the study. Index cases underwent routine clinical evaluation, including interrogation of family and medical history, physical examination, electrocardiogram (standard and high precordial leads), 24-h Holter, stress test, and had a yearly clinical examination for follow-up. Structural heart disease was ruled out by trans-thoracic echocardiography and/or magnetic resonance imaging. Informed consent was provided by participants or by their parents or legal guardian. The study was approved by the Ethics Committees of the National Institute of Cardiology Ignacio Chávez and the National Institute of Genomic Medicine in Mexico City.

The arrhythmogenic phenotypes considered were the following: SSS, sinus bradycardia, sinus pause or arrest with or without escape rhythm, sinoatrial exit block, tachy-brady syndrome, atrial fibrillation with slow ventricular response in the absence of AV node blocking agents, chronotropic incompetence [16]; PCCD: finding of a major conduction defect on the electrocardiogram (ECG) such as complete right bundle branch block, complete left bundle branch block, left anterior fascicular block / hemiblock or left posterior hemiblock, prolonged PR interval or complete AV block with broad QRS complexes [17]; $\mathrm{BrS}$, appearance of a type one ST-segment elevation (coved type) in more than one right precordial lead $\left(V_{1}\right.$ to $\left.V_{3}\right)$, in the presence or absence of a sodium channel blocker, and one of the following: documented ventricular fibrillation, ventricular tachycardia or selfterminating polymorphic ventricular tachycardia, a family history of sudden cardiac death 
(SCD, $<45$ years), coved type ECGs in family members, electrophysiological inducibility, syncope, or nocturnal agonal breathing [18]; LQTS was diagnosed when considered as high probability according the criteria of Schwartz [19]. Patients with more than one of these arrhythmogenic phenotypes were considered as SCN5A overlap syndrome cases.

\subsection{Targeted Sequencing}

Genomic DNA was extracted from peripheral blood of all participants using commercial methods (QIAGEN DNA Midi blood $\mathrm{kit}^{\circledR}$ ). Samples from all index cases were sequenced using the TruSight Cardio ${ }^{\circledR}$ Sequencing Panel on a Mi-Seq device (Illumina, San Diego, CA, USA). Post-run sequencing quality was assessed with FastQC (Babraham Bioninformatics, UK), reads were aligned with Burrows-Wheeler v2.0 (Broad Institute, Cambridge, MA, USA) [20]; variant calling was performed with the Genome Analysis Tool Kit (GATK v4.0; https: / / gatk.broadinstitute.org; accessed on 8 September 2021), and all variants were annotated with ANNOVAR (http://annovar.openbioinformatics.org; accessed on 8 September 2021).

All novel or very low frequency variants (minor allele frequency $<0.0005$ ) affecting the amino acid sequence of arrhythmogenic channelopathy-related genes were classified according to the American College of Medical Genetics and Genomics criteria as benign, likely benign, of unknown clinical significance (VUS), likely pathogenic (LP) or pathogenic. Patients with at least one pathogenic or likely pathogenic SCN5A variant and their available relatives were included in the analysis. Amino acid numbering was made according to transcription variant NM_198056 (http:/ / www.ncbi.nlm.nih.gov /, accessed on 8 September 2021).

\subsection{Statistical Analysis}

The numerical variables are expressed as mean $\pm \mathrm{SD}$ or median and interquartile range (IQR) as appropriate, and categorical variables as percentages. Overall penetrance for arrhythmogenic syndromes was estimated including all available individuals from families in which at least one first-degree relative was screened, as the ratio between number of SCN5A mutation carriers with arrhythmogenic phenotypes (affected) and the total number of carriers (affected and non-affected). Missense variants found in compound heterozygosity in probands, with no symptomatic heterozygous carriers in the family, were excluded from the overall penetrance estimation. Differences in SCN5A mutation penetrance between males and females were compared using the Chi-squared test.

\section{Results}

\subsection{SCN5A Mutations}

A total of 17 apparently unrelated patients with at least one pathogenic or likely pathogenic SCN5A variant were included in the study, along with a total of 42 firstand second-degree relatives. Table 1 summarizes the characteristics of all pathogenic or likely pathogenic SCN5A variants identified in the probands [21-29]. A total of sixteen independent variants were identified, twelve were missense, while four were inferred as null (three truncating frameshift and one a complex inframe multiple amino acid (aa) deletion/insertion occurring in a non-repetitive aa sequence in DIII/S5 (p.Trp1345Ser1349delinsPhe). All but three of the missense variants (p.Arg811Cys, p.Pro1730Leu and p.Ala1778Asp) had at least one functional study reporting loss and/or gain of function. Thirteen patients were heterozygous for missense, and one was heterozygous for a frameshift truncating mutation. Notably, three patients (17.6\%) were compound heterozygous for an inferred null mutation and a missense $S C N 5 A$ variant. Most missense mutations were located within the DIV domain $(6 / 12,50 \%)$, four $(33.3 \%)$ affected the pore forming region (three in DIV and one in DII), while two affected voltage sensor domains (S4) in DII and DIV. No other pathogenic or likely pathogenic variants were found among 174 cardiogenes in this group of patients. 
Table 1. SCN5A variants identified in children with arrhythmogenic syndromes.

\begin{tabular}{|c|c|c|c|c|c|c|c|}
\hline Variant & $\begin{array}{l}\text { Variant } \\
\text { Type }\end{array}$ & dbSNP & $\begin{array}{c}\text { gnomAD } \\
\text { Global MAF }\end{array}$ & $\begin{array}{l}\text { Domain } \\
\text { Location }\end{array}$ & Site & $\begin{array}{c}\text { Functional } \\
\text { Interpretation * }\end{array}$ & Reference \\
\hline p.Arg34fs * 60 & Frameshift & - & 0 & N-Terminus & - & LOF & [27] \\
\hline p.Val240Met & Missense & rs199473076 & $1.4 \times 10^{-5}$ & DI & $\begin{array}{l}\text { Cytoplasmic } \\
\text { S4-S5 }\end{array}$ & GOF & [22] \\
\hline p.Arg811Cys & Missense & rs794728864 & 0 & DII & S4 & - & - \\
\hline p.Arg893His & Missense & rs199473172 & $4.0 \times 10^{-6}$ & DII & Pore & LOF & [25] \\
\hline p.Arg1195His & Missense & rs199473596 & $2.5 \times 10^{-5}$ & $\begin{array}{l}\text { Interdomain } \\
\text { DII-DIII }\end{array}$ & Cytoplasmic & $\mathrm{LOF} / \mathrm{GOF}$ & [27] \\
\hline p.Asp1275Asn & Missense & rs137854618 & $8.0 \times 10^{-6}$ & DIII & S3 & LOF & [24] \\
\hline p.Trp1345_Ser1349delinsPhe & $\begin{array}{l}\text { Inframe } \\
\text { del/ins }\end{array}$ & & 0 & DIII & S5 & - & - \\
\hline p.Arg1632Cys & Missense & rs878855292 & $4.0 \times 10^{-6}$ & DIV & S4 & $\mathrm{LOF} / \mathrm{GOF}$ & [28] \\
\hline p.Arg1644His & Missense & rs199473282 & $4.0 \times 10^{-6}$ & DIV & $\begin{array}{l}\text { Cytoplasmic } \\
\text { S4-S5 }\end{array}$ & GOF & [26] \\
\hline p.Gly1661Arg & Missense & rs199473292 & 0 & DIV & S5 & LOF & [23] \\
\hline p.Thr1708Asn & Missense & - & 0 & DIV & Pore & LOF/GOF & [25] \\
\hline p.Ser1710Leu & Missense & rs137854604 & $1.6 \times 10^{-5}$ & DIV & Pore & LOF/GOF & [21] \\
\hline p.Pro1730Leu & Missense & rs1060501142 & $8 \times 10^{-6}$ & DIV & $\begin{array}{l}\text { Extracellular } \\
\text { pore }\end{array}$ & - & - \\
\hline p.Asp1741Glyfs * 48 & Frameshift & rs1251085820 & 0 & DIV S5/S6 & - & - & - \\
\hline p.Ala1778Asp & Missense & - & 0 & C-Terminus & Cytoplasmic & - & - \\
\hline p.Leu1821fs * 10 & Frameshift & rs794728924 & 0 & C-Terminus & Cytoplasmic & LOF/GOF & [29] \\
\hline
\end{tabular}

* Functional interpretation according to functional studies expressing the variants in different heterologous systems. MAF: minor allele frequency; LOF: loss of function; GOF: gain of function.

\subsection{Clinical Characteristics of Pediatric Arrhythmogenic Channelopathy Patients Bearing SCN5A Mutations}

Demographic and clinical characteristics of all index cases are described in Table 2. Fifteen of these patients $(88.2 \%)$ were male, and only two were female. Median age of symptoms onset was 6 years (IQR $=12$ years). Onset of symptoms occurred in the early childhood range $(<6$ years) in eight patients $(47.1 \%)$, during school age (6-12 years) in three patients $(17.6 \%)$, and during teenage years in six patients $(35.3 \%)$. Overall, the most prevalent arrhythmogenic syndrome was SSS (13/17, 76.5\%), followed by VT (64.7\%), PCCD (47.1\%), type $1 \mathrm{BrS}(35.3 \%)$ and LQTS (11.8\%). Notably, twelve patients $(70.6 \%)$ had overlap syndrome, two $(11.8 \%)$ had isolated SSS, two more had isolated $\mathrm{BrS}$, one $(5.9 \%)$ had isolated type 3 LQTS. Considering only patients with overlap syndrome, the most prevalent phenotypes were SSS and VT (11/12, 91.7\% each), followed by PCCD (66.7\%), type $1 \mathrm{BrS}(33.3 \%)$ and LQTS (8.3\%). Pacemakers were implanted in all patients with SSS, and an implantable cardioverter defibrillator was implanted in the only patient who had suffered a ventricular fibrillation event. Figure 1 depicts mutation sites and ECGs from all probands.

Three apparently unrelated patients were heterozygous for the p.Ser1710Leu mutation, each with a different phenotype: overlap syndrome (SSS and PCCD), isolated SSS and isolated BrS. Two apparently unrelated patients were p.Arg893His heterozygous, one with overlap and the other with isolated BrS. In addition, two apparently unrelated children were heterozygous for the p.Thr1708Asn mutation, both with overlap syndrome and schoolaged at the onset of symptoms. On further interrogation, family members were able to identify that both index cases were in fact second cousins once removed. 
A)

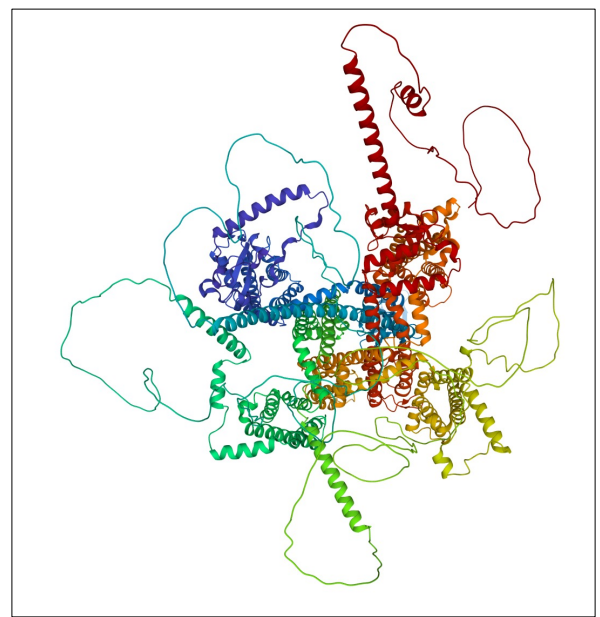

B)

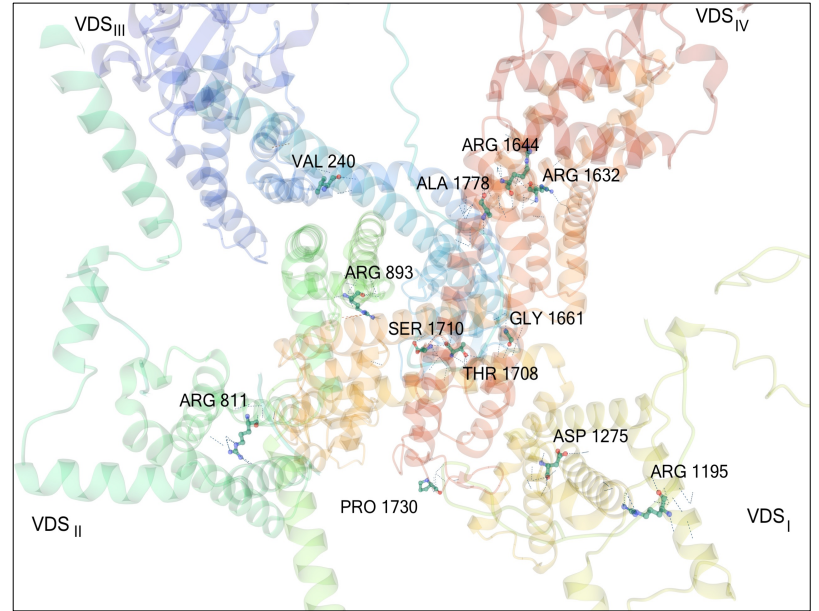

C)

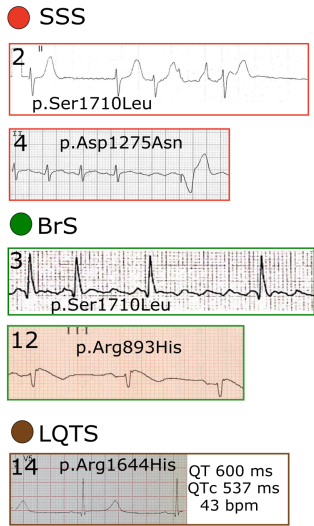

SSS, PCCD

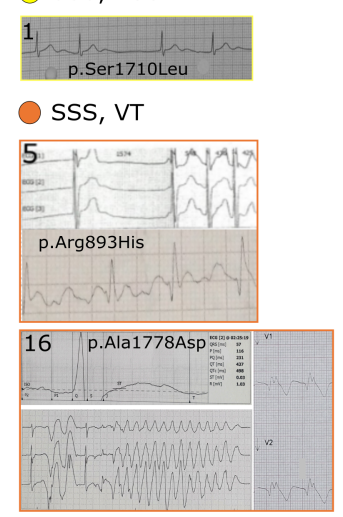

VT, LQTS

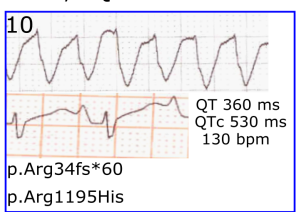

SSS, PCCD, VT

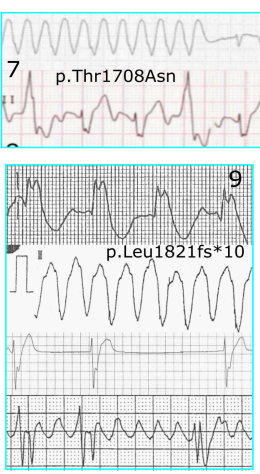

SSS, VT, BrS

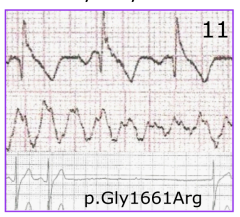

SSS, PCCD, VT, BrS
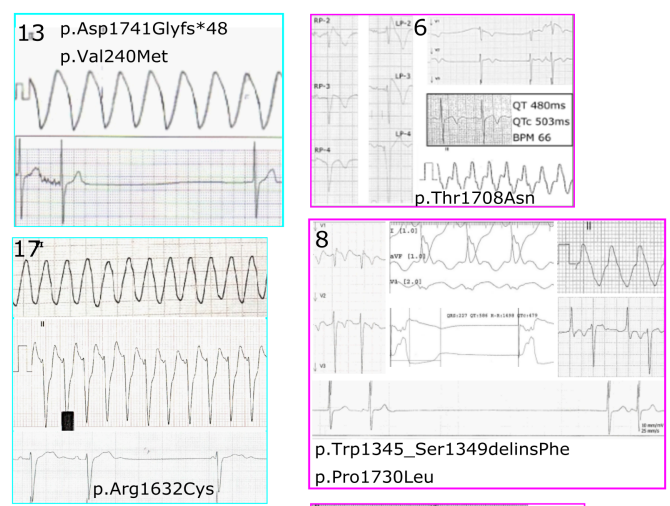

15

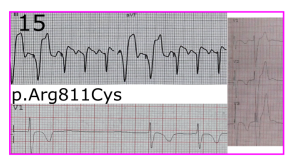

Figure 1. Molecular mapping of the SCN5A mutations. (A) Cartoon representation of the overall structure of Nav1.5: DI, DII, DIII and DIV domains are colored yellow, green, blue and red, respectively. (B) Upper view of the cryo-EM reconstruction of $\mathrm{Na}_{\mathrm{V}} 1.5$ using the UniProt (https://www.uniprot.org/uniprot/Q14524, accessed on 28 October 2021) and $\alpha$ Fold templates. SCN5A mutations are indicated as balls and sticks. (C) Representative ECGs of probands are presented. The case number is indicated on the ECG image. Cases are ordered according to arrhythmogenic phenotypes, SSS: sick sinus syndrome; PCCD: progressive cardiac conduction disease; VT: ventricular tachycardia; BrS: Brugada syndrome; LQTS: long QT syndrome. ECG from Case 2: atrial standstill; Case 4: atrial flutter with VVI pacemaker escape; Case 3: atrial flutter; Case 12: coved type ST elevation and sinus bradycardia; Case 14: sinus bradycardia with prolonged QTc interval (537 ms); Case 1: sinus bradycardia; Case 5: sinus standstill with monomorphic ventricular tachycardia; Case 16: sinus standstill with ventricular fibrillation; Case 10: monomorphic ventricular tachycardia; Case 7: sustained monomorphic ventricular tachycardia; Case 9: severe intraventricular conduction disease, monomorphic ventricular tachycardia, sinus standstill and atrial flutter; Case 13: sustained monomorphic ventricular tachycardia and sinus standstill; Case 17: monomorphic ventricular tachycardia and atrial standstill; Case 11: sinus bradycardia, ventricular fibrillation and sinus standstill; Case 6: sinus standstill, prolonged QTc interval $(507 \mathrm{~ms})$ and sustained monomorphic ventricular tachycardia; Case 8: severe cardiac intraventricular conduction disease, sustained monomorphic ventricular tachycardia and sinus standstill; Case 15: sinus standstill and VVI pacemaker. 
Table 2. Clinical characteristics of SCN5A-channelopathy pediatric patients.

\begin{tabular}{|c|c|c|c|c|c|c|c|c|c|c|c|c|c|c|c|}
\hline Case & Variant & Sex & $\begin{array}{l}\text { Age of } \\
\text { Onset }\end{array}$ & Syncope & Seizures & Device & NFCA & SCD & $\begin{array}{l}\text { Arrhythmogenic } \\
\text { Phenotype }\end{array}$ & SVT & VA & AVB & $\begin{array}{l}\text { RBBB, } \\
\text { LBBB }\end{array}$ & AS & $\underset{(\mathrm{ms})}{\operatorname{MaxQTc}}$ \\
\hline 1 & p.Ser1710Leu & $\mathrm{M}$ & 14 & - & - & PM & - & - & SSS, PCCD & $\begin{array}{l}\text { aFL, } \\
\text { CTI-D }\end{array}$ & - & $3 \mathrm{rd} D$ & - & - & $488^{*}$ \\
\hline 2 & p.Ser1710Leu & $\mathrm{M}$ & 2 & Yes & - & PM & - & - & sss & - & - & - & RBBB & - & $682^{*}$ \\
\hline 3 & p.Ser1710Leu & M & 15 & - & - & - & - & - & $\mathrm{BrS}$ & $\begin{array}{l}\text { AF, aFL, } \\
\text { CTI-D }\end{array}$ & - & 1st D & - & - & 413 \\
\hline 4 & p.Asp1275Asn & M & 17 & - & - & $\mathrm{PM}$ & - & - & SSS & - & - & 3rd D & $\begin{array}{l}\text { RBBB, } \\
\text { LBBB }\end{array}$ & Yes & $476^{*}$ \\
\hline 5 & p.Arg893His & M & 15 & Yes & - & PM & - & - & SSS, VT & $\begin{array}{l}\text { aFL, } \\
\text { CTI-D }\end{array}$ & SMVT & $3 r d D$ & RBBB & Yes & $542 *$ \\
\hline 6 & p.Thr1708Asn & $\mathrm{M}$ & 6 & Yes & - & PM & - & - & $\begin{array}{l}\text { SSS, PCCD, VT, } \\
\text { BrS }\end{array}$ & $\begin{array}{l}\text { AF, aFL, } \\
\text { AT }\end{array}$ & SMVT & - & $\begin{array}{l}\text { RBBB, } \\
\text { LBBB }\end{array}$ & - & $503^{*}$ \\
\hline 7 & p.Thr1708Asn & M & 10 & - & - & PM & - & Yes & SSS, PCCD, VT & aFL & SMVT & 1st D & $\begin{array}{l}\text { RBBB, } \\
\text { LBBB }\end{array}$ & - & 481 * \\
\hline 8 & $\begin{array}{l}\text { p.Trp1345_Ser } \\
\text { 1349delinsPhe/ } \\
\text { p.Pro1730Leu }\end{array}$ & F & 3 & Yes & - & PM & Yes & - & $\begin{array}{l}\text { SSS, PCCD, VT, } \\
\text { BrS }\end{array}$ & $\mathrm{AF}, \mathrm{aFL}$ & $\begin{array}{l}\text { SMVT, } \\
\text { PVT }\end{array}$ & $3 \mathrm{rd} \mathrm{D}$ & $\begin{array}{l}\text { RBBB, } \\
\text { LBBB }\end{array}$ & Yes & 495 * \\
\hline 9 & p.Leu1821fs ${ }^{*} 10$ & M & 12 & - & - & PM & Yes & Yes & SSS, PCCD, VT & aFL & SMVT & 2nd D & RBBB & - & 550 * \\
\hline 10 & $\begin{array}{l}\text { p.Arg34fs *60/ } \\
\text { p.Arg1195His }\end{array}$ & $\mathrm{M}$ & $22 \mathrm{mo}$ & Yes & Yes &.- & Yes & Yes & VT, LQTS & - & $\begin{array}{l}\text { SMVT, } \\
\text { VF }\end{array}$ & - & - & - & 519 \\
\hline 11 & p.Gly1661Arg & M & 2 & Yes & Yes & ICD & Yes & - & SSS, VT, BrS & - & $\begin{array}{l}\text { SMVT, } \\
\text { VF }\end{array}$ & - & RBBB & - & $500 *$ \\
\hline 12 & p.Arg893His & M & 17 & Yes & - & - & Yes & Yes & $\mathrm{BrS}$ & - & $\mathrm{VF}$ & - & - & - & 402 \\
\hline 13 & $\begin{array}{c}\text { p.Asp1741Glyfs } \\
{ }^{*} 48 / \\
\text { p.Val240Met }\end{array}$ & $\mathrm{M}$ & 4 & Yes & - & PM & - & - & SSS, PCCD, VT & $\mathrm{AF}$ & SMVT & 1st D & RBBB & - & $630 *$ \\
\hline 14 & p.Arg1644His & F & 16 & Yes & - & - & - & - & LQTS & АT & - & - & - & - & 520 \\
\hline 15 & p.Arg811Cys & M & 4 & - & - & PM & - & - & $\begin{array}{l}\text { SSS, PCCD, VT, } \\
\text { BrS }\end{array}$ & $\mathrm{aFL}$ & SMVT & $3 \mathrm{rd} \mathrm{D}$ & $\begin{array}{l}\text { RBBB, } \\
\text { LBBB }\end{array}$ & Yes & $519 *$ \\
\hline 16 & p.Ala1778Asp & $\mathrm{M}$ & 4 & Yes & Yes & - & - & - & SSS, VT & $\mathrm{AF}$ & $\begin{array}{l}\text { PVT, } \\
\text { TdP }\end{array}$ & 1st D & RBBB & Yes & $529 *$ \\
\hline 17 & p.Arg1632Cys & M & 3 & - & Yes & PM & - & Yes & SSS, PCCD, VT & $\mathrm{AF}$ & SMVT & - & $\begin{array}{l}\text { RBBB, } \\
\text { LBBB }\end{array}$ & - & $462^{*}$ \\
\hline
\end{tabular}

NFCA: Non-fatal cardiac arrest; SCD: Sudden cardiac death; SVT: Supraventricular tachycardia; VA: Ventricular arrhythmia; AVB: Atrioventricular block; RBBB: Right bundle branch block; LBBB: Left bundle branch block; AS: Atrial standstill; MaxQTc: maximum corrected QT interval; SSS: Sick sinus syndrome; PCCD: Progressive cardiac conduction disease; VT: idiopathic ventricular tachycardia; BrS: Brugada syndrome; LQTS: long QT syndrome; PM: pacemaker; ICD: Implantable cardioverter defibrillator; AF: Atrial fibrillation; aFL: Atrial flutter; CTI-D: cavotricuspid isthmus-dependent atrial flutter. AT: atrial tachycardia; SMVT: Sustained monomorphic ventricular tachycardia; PVT: polymorphic ventricular tachycardia; FV: ventricular fibrillation; TdP: Torsade de points. * Prolonged QTc interval secondary to intraventricular conduction defect or AV block. Cases 9 and 10 were previously published $[27,29]$.

\subsection{Syncope, Seizures, Non-Fatal Cardiac Arrest (NFCA) and Sudden Cardiac Death (SCD)}

Syncope was reported in ten (58.8\%) patients, and seizures in four (23.5\%). The frequency of non-fatal cardiac arrest was $29.4 \%(5 / 17)$, and three of these five patients suffered SCD afterwards. The overall frequency of sudden cardiac death was also $29.4 \%$, occurring before age 12 in 4 patients with overlap syndrome, and at age 17 in an isolated BrS patient.

\subsection{ECG and Holter Findings}

Twelve patients had at least one type of supraventricular tachycardia (SVT). The most prevalent was atrial flutter $(8 / 17,47.1 \%)$, followed by atrial fibrillation $(35.3 \%)$, cavotricuspid isthmus-dependent atrial flutter $(17.6 \%)$ and atrial tachycardia $(11.8 \%)$. Moreover, twelve patients had one or more documented ventricular tachycardia events; the most prevalent was sustained monomorphic ventricular tachycardia $(58.8 \%)$, followed by ventricular fibrillation (17.6\%), polymorphic ventricular tachycardia $(11.8 \%)$; and torsade de points (5.9\%); ten had atrio-ventricular block (58.8\%) and five had documented atrial standstill $(29.4 \%)$. Finally, $15 / 17$ patients $(76.5 \%)$ showed a prolonged QTc interval (QTc max $>460 \mathrm{~ms}$ ), which was likely secondary to intraventricular conduction defect or AV block in 13 , and only two patients had a normal maximum QTc interval $(<440 \mathrm{~ms})$.

\subsection{Incomplete Penetrance and Variable Expressivity}

Figures 2 and 3 show family pedigrees of index cases with autosomal dominant inheritance (heterozygous mutations), where at least one parent was available for clinical assessment and DNA analysis (all except cases 4, 5, 11 and 17), showing incomplete 
penetrance and variable expressivity. Different phenotypes were observed both for the same mutation in different cases (cases 1, 2 and 3 for p.Ser1710Leu; cases 6 and 7 for p.Thr1708Asn), and within the same family (pedigrees for cases 6, 7 and 9). Case 14 was the only isolated LQTS case, she and her father were heterozygous for the p.Arg1644His mutation; however, her father's ECG showed a normal QTc interval (432 ms) with bradycardia (49 bpm). From a total of 45 SCN5A mutation carriers, 24 were affected with an arrhythmogenic phenotype (overall penetrance $53.3 \%$ ), and penetrance was higher in males $(63.3 \%, 19$ affected $/ 30$ carriers) than in females (33.3\%, five affected $/ 15$ carriers; $p=0.057$ ).

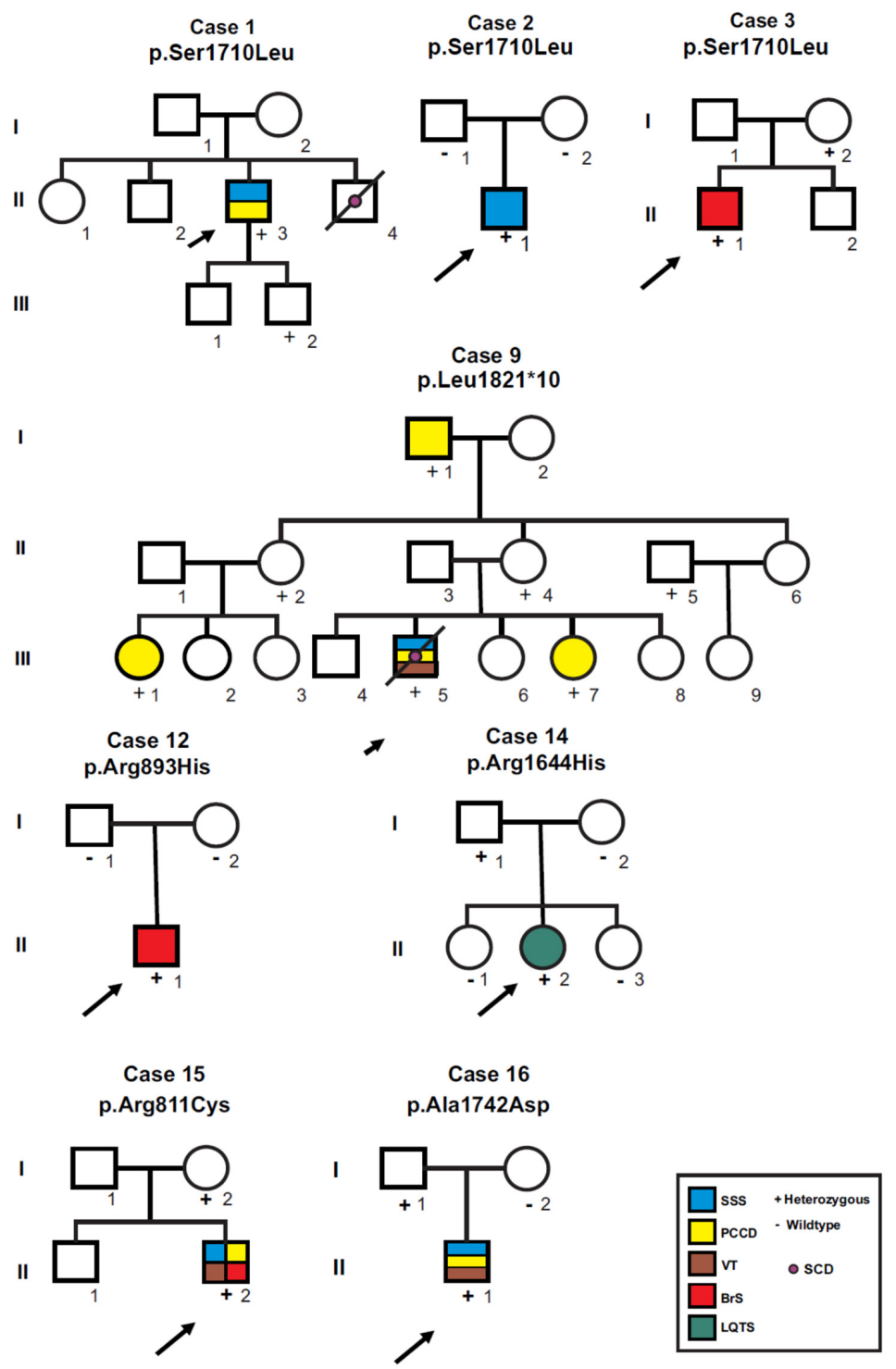

Figure 2. Pedigrees of probands heterozygous for SCN5A mutations where at least one first-degree relative was available for screening. Circles indicate females, squares, males; symbols with diagonal lines represent deceased individuals. Open symbols represent asymptomatic individuals, filled symbols were affected by arrhythmogenic syndromes according to the color code. Parentship was not confirmed by genetic analyses in any case. SSS: sick sinus syndrome; PCCD: progressive cardiac conduction disease; VT: ventricular tachycardia; BrS: Brugada syndrome; LQTS: long QT syndrome; SCD: sudden cardiac death. 


\section{p.Thr1708Asn}

I

II

III

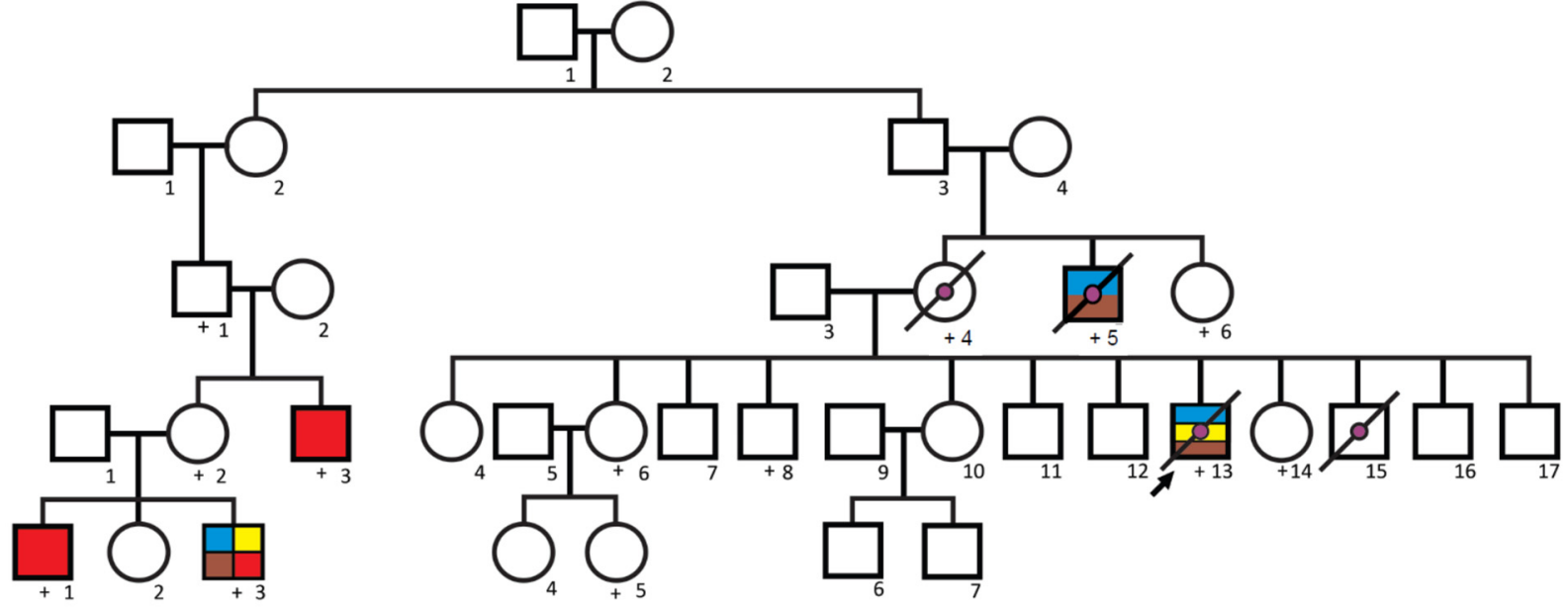

V

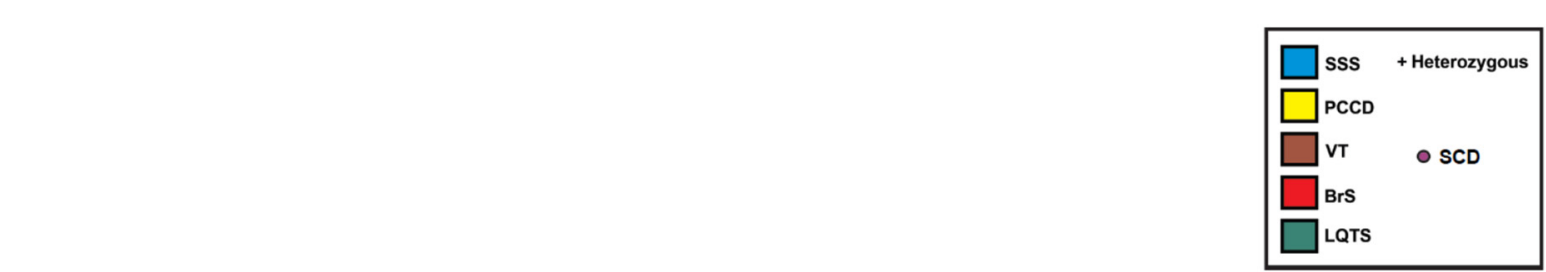

Figure 3. Pedigree of the family bearing the p.Thr1708Asn mutation. Circles indicate females, squares, males. Symbols with diagonal lines represent deceased individuals. Open symbols represent asymptomatic individuals, filled symbols are affected by arrhythmogenic syndromes according to the color code. Case 6 (V-3) and case 7 (IV-13) were initially thought to be unrelated, but after thorough interrogation were found to be first cousins once removed. Individual III-4 suffered sudden cardiac death at age 39 years, III- 5 at age 56 years; and IV-15 at age 6 years. p.Thr1708Asn heterozygous individuals III-6, IV-6, IV-8, and V-5 had minor ECG manifestations, including prolonged PR and/or prolonged QTC intervals. SSS: sick sinus syndrome; PCCD: progressive cardiac conduction disease; VT: ventricular tachycardia; BrS: Brugada syndrome; LQTS: long QT syndrome; SCD: sudden cardiac death.

Penetrance of individual mutations was estimated when five or more heterozygous carriers were found. A total of 14 individuals of a single kindred carried the p.Thr1708Asn mutation (eight male and six female); penetrance for arrhythmogenic phenotypes was $6 / 8(75.0 \%)$ in males (three with overlap syndrome, two with BrS and one with sudden cardiac death at age 6). No arrhythmic phenotypes were observed in available female carriers, but $3 / 4$ (75\%) showed minor ECG abnormalities such as AV block ( $\mathrm{PR}<200 \mathrm{~ms})$ and/or prolonged QTc interval (>470 ms, range 478-488 ms). Moreover, a total of seven individuals from a single kindred carried the p.Leu1821fs*10 mutation. Four were female (two with PCCD and two asymptomatic) and three were male (one with PCCD, one with overlap syndrome and one asymptomatic). Finally, five individuals from three different kindreds were heterozygous for the p.Ser1710Leu mutation, an asymptomatic female and four males (one asymptomatic, one each with overlap syndrome, PCCD and BrS).

\subsection{Compound Heterozygosity for SCN5A Mutations}

Figure 4 shows the pedigrees of the three index cases (17.6\%) found to be compound heterozygous for SCN5A mutations, with an onset of symptoms in early childhood (younger than age 4 years), overlap syndrome and prolonged QTc intervals. Case 8 (p.Trp1345_Ser1349delinsPhe/ p.Pro1730Leu) was a severely affected six year-old girl who showed episodes of palpitations, fatigue and documented bradycardia since age three years, and was diagnosed with SSS, PCCD, VT, type 1 BrS and showed a prolonged QTC 
interval (495ms). During hospital stay she suffered various arrhythmic events including atrial flutter, atrial standstill, sustained ventricular tachycardia, ventricular fibrillation, and notably, bidirectional ventricular tachycardia and two cardiac arrest episodes with successful reanimation after cardio-pulmonary resuscitation. Both parents were asymptomatic, however a p.Trp1345_Ser1349delinsPhe heterozygous paternal first cousin showed a type 1 BrS ECG pattern. In addition, four asymptomatic relatives (II-7, III-5, III-6 and IV-3) carried the p.Pro1730Leu variant.

Case 8

p.Trp1345_Ser1349delinsPhe/p.Pro1730Leu

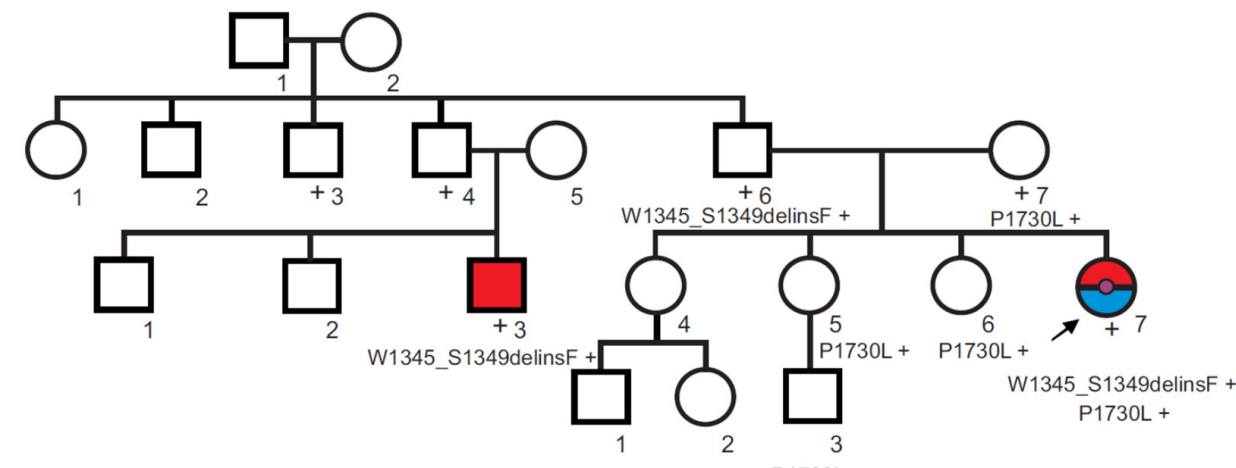

Case 13 p.Asp1741Glyfs ${ }^{*} 48 /$ p.Val240Met

I

II

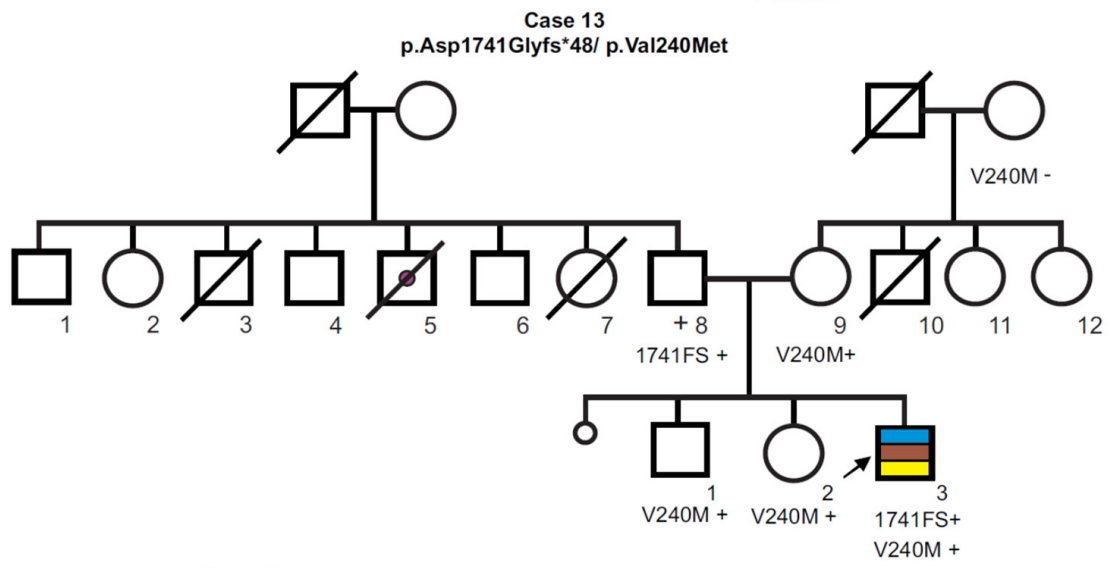

Case 10

p.Arg34fs ${ }^{*} 60 /$ p.Arg1195His

I

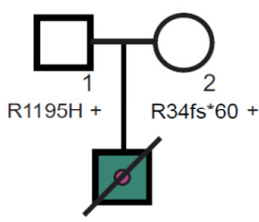

$\mathrm{R} 1195 \mathrm{H}+$

$\mathrm{R} 34 \mathrm{fs}^{*} 60+$

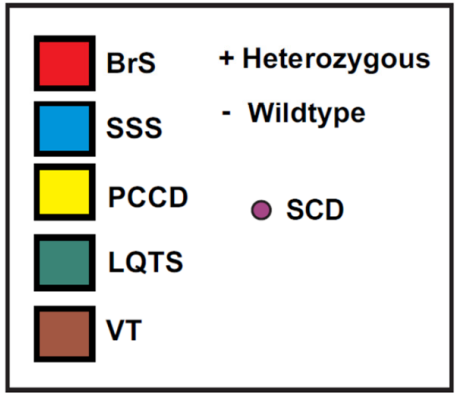

Figure 4. Pedigree of 3 overlap syndrome cases who were compound heterozygous for SCN5A mutations. Circles indicate females, squares, males; symbols with diagonal lines represent deceased individuals. Open symbols represent asymptomatic individuals, filled symbols were affected by arrhythmogenic syndromes according to the color code. SSS: sick sinus syndrome; PCCD: progressive cardiac conduction disease; VT: ventricular tachycardia; BrS: Brugada syndrome; LQTS: long QT syndrome; SCD: sudden cardiac death. 
Case 10 (p.Arg34fs*60/p.Arg1195His) was a 22 month-old male toddler [27], who was admitted to the emergency room with febrile seizures and syncope and was diagnosed with SSS and VT, and a prolonged QTc interval (maximum QTc $=519 \mathrm{~ms}$ ) with no apparent intraventricular conduction defect. The boy showed signs of severe neurological injury and died shortly after being admitted. He inherited the p.Arg1195His variant from his father, the p.Arg34fs*60 variant from his mother, and both parents were asymptomatic. Finally, case 13 (p.Asp1741Glyfs*48/p.Val240Met) presented with syncope at age 4 years, and was diagnosed with SSS, PCCD and recurrent monomorphic VT. The QT interval on ECG was prolonged (QTc $=630 \mathrm{~ms}$ ). The mother (II-9) and two siblings (III-1 and III-2) had the Val240Met variant and were asymptomatic, while the father was p.Asp $1741 \mathrm{Glyfs} * 48$ heterozygous and was also asymptomatic.

\section{Discussion}

The field of cardiovascular genetics is rapidly evolving and has greatly contributed to the understanding of primary electrical heart disease. However, this field faces an enormous challenge when it comes to the integration of genetics, functional studies, diagnosis, prognosis, and management [30]. The growing list of SCN5A channelopathy and overlap syndrome reports in the medical literature confirms that incomplete penetrance, male predominance and variable expressivity are characteristic of the disease, attributed to both genetic and non-genetic factors [7]. In the present study, all families with more than one SCN5A mutation carrier showed this variable expressivity, involving differences in age of onset (childhood-adulthood), arrhythmogenic phenotypes and disease severity. Similarly, apparently unrelated patients sharing the same mutation (Cases 1, 2 and 3 for p.Ser1710Leu) also showed variable expressivity regarding arrhythmogenic phenotypes. As expected, overall penetrance and penetrance of individual mutations was higher in male than in female individuals.

To our knowledge only one prospective study of children with SCN5A mutations included 442 neonates, where most showed no ECG alterations at birth $(44.3 \%)$ and the most frequent arrhythmogenic phenotype was isolated PCCD (25.6\%), followed by overlap syndrome (15\%), LQTS (10.6\%) and BrS (1.8\%) [15]. The present study includes only pediatric patients who were symptomatic and required specialized medical assistance at the Ignacio Chávez National Institute of Cardiology. In agreement with the study in neonates, isolated LQTS and isolated BrS were the least frequent phenotypes (5.9\% each), however among the symptomatic children of the present study, the most frequent phenotype was overlap syndrome (70.6\%).

The high frequency of children with compound heterozygous SCN5A mutations in our group of symptomatic children $(17.6 \%)$ is noteworthy. Compound heterozygosity is infrequent in SCN5A channelopathies, reported only in $13 / 2111(0.62 \%)$ of BrS patients [31], and in $0.7 \%$ of neonates with SCN5A mutations [15]. Moreover, among ten individual SCN5A compound heterozygous case reports [12,13,27,32-38], only two cases reported adult age of onset $[35,36]$, and $6 / 8$ childhood onset cases had a truncating mutation combined with a missense mutation [12,27,32-34]. All three of our compound heterozygous cases (cases 8, 10 and 13) carried an inferred null and a missense mutation, had a severe overlap syndrome with early childhood onset ( $<4$ years), and NFCA events occurred in two of these cases. Notably, case 8 had documented polymorphic ventricular tachycardia and bidirectional ventricular tachycardia, considered a calcium-handling arrhythmia. To our knowledge, there is only one report of a SCN5A mutation (p.T1857I) in a family with multiple sudden cardiac deaths where afflicted probands presented with atrial and ventricular arrhythmias including bidirectional VT [39]. Functional studies in heterologous cells and the use of O'Hara Rudy and Grandi models [40,41] suggested the mutation caused right-shifted voltage-dependence of both activation (gain of function, GOF) and inactivation (loss of function, LOF), with a net gain-of-function in the $\mathrm{Na}_{V} 1.5$ gating, an increased window current and resultant ventricular tissue after depolarization. 
Among the genetic modifiers contributing to variable expressivity and incomplete penetrance, studies in heterologous cells have shown common SCN5A polymorphisms p.H558R and p.del1077Q not only affect electrophysiological properties of $\mathrm{Na}_{\mathrm{V}} 1.5$, but may also modulate the effects of co-existing disease-causing mutations [42-44]. In this regard, a functional study in HEK293 cells showed that the p.Thr1708Asn mutation decreased the peak $\mathrm{I}_{\mathrm{Na}}$ current and increased the late $\mathrm{I}_{\mathrm{NaL}}$ current, decreasing the peak $\mathrm{I}_{\mathrm{Na}}$ current even further when found in cis with the p.H558R polymorphism [25]. Segregation analysis of the p.Thr1708Asn kindred (Figure 3) showed that both variants were in fact on the same allele, as two of the p.Thr1708Asn carriers (IV-13 and IV-14) were p.558R homozygous (data not shown), compatible with the severe phenotype observed in several affected males. Unfortunately, the number of individuals with SCN5A mutations was insufficient to assess the possible association of common polymorphisms with clinical severity in our series.

The consequences of most of the mutations identified in this group of patients have been studied in heterologous ion channel expression systems [16-24]. However, because of the many consequences of mutations on electrophysiological phenotypes (early and late inward Na currents, activation or inactivation properties, interactions with other proteins, response to adrenergic or cholinergic stimuli, response to anti-arrhythmic drugs, etc.), and because different functional phenotypes often coexist, labeling variants as GOF and/or LOF may be an oversimplification. Particularly, because it has been proposed that $\mathrm{Na}_{\mathrm{V}} 1.5$ channels assemble as dimers [3], compound heterozygous mutations may require the biophysical characterization of both mutations separately and in coexpression. In this regard, previous functional studies of the mutations of case 10 showed the frame-shifted and prematurely truncated peptide SCN5A-Arg $34 \mathrm{fs}^{*} 60$ produced no current, while SCN5A-Arg1195His had a normal peak and late current but abnormal voltage-dependent gating parameters. Interestingly, co-expression of both variants led to a significant increase in late $\mathrm{I}_{\mathrm{NaL}}$ current [27]. Functional studies of SCN5A variants found in case 8 (p.Trp1345_Ser1349delinsPhe/p.Pro1730Leu) and in case 13 (p.Asp1741Glyfs*48/ p.Val240Met), both individually and in co-expression, are pending, as are functional studies of three of the missense variants here identified (p.Arg811Cys; p.Pro1730Leu, p.Ala1778Asp). While these models have a number of shortcomings, as they do not closely reproduce human heart physiology and clinical manifestations [45,46], functional and pharmacological studies in induced pluripotent cell (iPSC)-derived cardiomyocytes have overcome several of these shortcomings and have become a promising option for precision medicine [47]. These functional studies, together with careful and detailed clinical characterization of patients and apparently asymptomatic mutation carriers, are crucial to understand the complexities of SCN5A channelopathies.

In conclusion, SCN5A-disease associated phenotypes occurring in the pediatric age were characterized mainly by overlap syndrome, sick sinus syndrome, cardiac conduction disease and ventricular tachycardia. It is important to recognize these phenotypes as potential markers of sodium channel disease, and to avoid the use of sodium channel blockers which can exacerbate the phenotype or even cause irreversible ventricular arrhythmias in the setting of pathogenic variants affecting SCN5A.

Author Contributions: Conceptualization, T.V.-M., A.M.-D. and P.I.; Methodology, G.P.G.-O., Á.E.R.-Q., M.D.-P., L.J.-A. and S.N.; validation, G.P.G.-O., Á.E.R.-Q., M.D.-P. and L.J.-A.; investigation, T.V.-M., G.P.G.-O., Á.E.R.-Q., M.D.-P., L.J.-A., S.N., A.C., A.M.-D. and P.I.; resources, T.V.-M., A.C., A.M.-D. and P.I.; data curation, G.P.G.-O., L.J.-A. and M.D.-P.; writing-original draft preparation, T.V.-M.; writing-review and editing, T.V.-M., M.D.-P., L.J.-A., A.C., A.M.-D., P.I.; visualization, G.P.G.-O., Á.E.R.-Q., M.D.-P. and L.J.-A.; supervision, S.N., T.V.-M., A.M.-D. and P.I.; project administration, T.V.-M. and P.I.; funding acquisition, T.V.-M., A.C., A.M.-D. and P.I. All authors have read and agreed to the published version of the manuscript.

Funding: This research was partially funded by Consejo Nacional de Ciencia y Tecnología (CONACyT), Cátedras-CONACyT grant number 1703, FOSISS grant numbers 261670 and SALUD-2010-01140259. Gabriela Paola García Ordóñez is a postgraduate student from Posgrado en Ciencias Médicas, 
Odontológicas y de la Salud, ICES, Bioquímica Clínica, Universidad Nacional Autónoma de México UNAM and received fellowship number 719803 from CONACyT.

Institutional Review Board Statement: The study was conducted according to the guidelines of the Declaration of Helsinki and approved by the Institutional Research and Ethics Committees of Instituto Nacional de Medicina Genómica (protocol code 03/2011/I approved on 22 February 2011, and protocol code 06/2014/I approved on 11 August 2014). It was also approved by the Research and Ethics Committees of the Instituto Nacional Cardiología "Ignacio Chávez" (protocol code 11-727 approved on 16 August 2011, and protocol code number 18-1053 approved on 22 May 2018).

Informed Consent Statement: Informed consent was obtained from all subjects involved in the study or their parents or legal guardians.

Data Availability Statement: Data available on request from the corresponding authors, due to restrictions on patient privacy as established in the informed consent.

Acknowledgments: Alvaro E. Reyes-Quintero wishes to thank the Mexican Society of Cardiology (Sociedad Mexicana de Cardiología) for their financial support with a scholarship during his stay as research assistant at the Department of Electrocardiology at the National Institute of Cardiology Ignacio Chávez.

Conflicts of Interest: The authors declare no conflict of interest. The funders had no role in the design of the study; in the collection, analyses, or interpretation of data; in the writing of the manuscript, or in the decision to publish the results.

\section{References}

1. Veerman, C.C.; Wilde, A.A.; Lodder, E.M. The cardiac sodium channel gene SCN5A and its gene product Nav1.5: Role in physiology and pathophysiology. Gene 2015, 573, 177-187. [CrossRef]

2. Shy, D.; Gillet, L.; Abriel, H. Cardiac sodium channel Nav1.5 distribution in myocytes via interacting proteins: The multiple pool model. Biochim. Biophys. Acta 2013, 1833, 886-894. [CrossRef]

3. Clatot, J.; Hoshi, M.; Wan, X.; Liu, H.; Jain, A.; Shinlapawittayatorn, K.; Marionneau, C.; Ficker, E.; Ha, T.; Deschenes, I. Voltage-gated sodium channels assemble and gate as dimers. Nat. Commun. 2017, 8, 2077. [CrossRef]

4. Remme, C.A. Cardiac sodium channelopathy associated with SCN5A mutations: Electrophysiological, molecular and genetic aspects. J. Physiol. 2013, 591, 4099-4116. [CrossRef]

5. Ruan, Y.; Liu, N.; Priori, S.G. Sodium channel mutations and arrhythmias. Nat. Rev. Cardiol. 2009, 6, 337-348. [CrossRef]

6. Wilde, A.A.M.; Amin, A.S. Clinical Spectrum of SCN5A Mutations: Long QT Syndrome, Brugada Syndrome, and Cardiomyopathy. JACC Clin. Electrophysiol. 2018, 4, 569-579. [CrossRef]

7. Verkerk, A.O.; Amin, A.S.; Remme, C.A. Disease Modifiers of Inherited SCN5A Channelopathy. Front. Cardiovasc. Med. 2018, 5, 137. [CrossRef]

8. Blich, M.; Khoury, A.; Suleiman, M.; Lorber, A.; Gepstein, L.; Boulous, M. Specific Therapy Based on the Genotype in a Malignant Form of Long QT3, Carrying the V411M Mutation. Int. Heart J. 2019, 60, 979-982. [CrossRef]

9. Chockalingam, P.; Rammeloo, L.A.; Postema, P.G.; Hruda, J.; Clur, S.A.; Blom, N.A.; Wilde, A.A. Fever-induced life-threatening arrhythmias in children harboring an SCN5A mutation. Pediatrics 2011, 127, e239-e244. [CrossRef]

10. Kilinc, O.U.; Tuzcu, V. Successful elimination of significant arrhythmia burden with flecainide in an adolescent with long QT syndrome type 3. Congenit. Heart Dis. 2012, 7, E42-E45. [CrossRef]

11. Kwon, H.W.; Lee, S.Y.; Kwon, B.S.; Kim, G.B.; Bae, E.J.; Kim, W.H.; Noh, C.I.; Cho, S.I.; Park, S.S. Long QT syndrome and dilated cardiomyopathy with SCN5A p.R1193Q polymorphism: Cardioverter-defibrillator implantation at 27 months. Pacing Clin. Electrophysiol. 2012, 35, e243-e246. [CrossRef]

12. Nijak, A.; Labro, A.J.; De Wilde, H.; Dewals, W.; Peigneur, S.; Tytgat, J.; Snyders, D.; Sieliwonczyk, E.; Simons, E.; Van Craenenbroeck, E.; et al. Compound Heterozygous SCN5A Mutations in Severe Sodium Channelopathy with Brugada Syndrome: A Case Report. Front. Cardiovasc. Med. 2020, 7, 117. [CrossRef]

13. Sacilotto, L.; Epifanio, H.B.; Darrieux, F.C.; Wulkan, F.; Oliveira, T.G.; Hachul, D.T.; Pereira, A.D.; Scanavacca, M.I. Compound Heterozygous SCN5A Mutations in a Toddler-Are they Associated with a More Severe Phenotype? Arq. Bras. Cardiol. 2017, 108, 70-73. [CrossRef]

14. Tsukakoshi, T.; Lin, L.; Murakami, T.; Shiono, J.; Izumi, I.; Horigome, H. Persistent QT Prolongation in a Child with Gitelman Syndrome and SCN5A H558R Polymorphism. Int. Heart J. 2018, 59, 1466-1468. [CrossRef] [PubMed]

15. Baruteau, A.E.; Kyndt, F.; Behr, E.R.; Vink, A.S.; Lachaud, M.; Joong, A.; Schott, J.J.; Horie, M.; Denjoy, I.; Crotti, L.; et al. SCN5A mutations in 442 neonates and children: Genotype-phenotype correlation and identification of higher-risk subgroups. Eur. Heart J. 2018, 39, 2879-2887. [CrossRef]

16. De Ponti, R.; Marazzato, J.; Bagliani, G.; Leonelli, F.M.; Padeletti, L. Sick Sinus Syndrome. Card. Electrophysiol. Clin. 2018, 10, 183-195. [CrossRef] [PubMed] 
17. Baruteau, A.E.; Probst, V.; Abriel, H. Inherited progressive cardiac conduction disorders. Curr. Opin. Cardiol. 2015, 30, 33-39. [CrossRef] [PubMed]

18. Brugada, J.; Campuzano, O.; Arbelo, E.; Sarquella-Brugada, G.; Brugada, R. Present Status of Brugada Syndrome: JACC State-of-the-Art Review. J. Am. Coll. Cardiol. 2018, 72, 1046-1059. [CrossRef]

19. Schwartz, P.J.; Ackerman, M.J. The long QT syndrome: A transatlantic clinical approach to diagnosis and therapy. Eur. Heart J. 2013, 34, 3109-3116. [CrossRef]

20. Li, H.; Durbin, R. Fast and accurate long-read alignment with Burrows-Wheeler transform. Bioinformatics 2010, 26, 589-595. [CrossRef]

21. Akai, J.; Makita, N.; Sakurada, H.; Shirai, N.; Ueda, K.; Kitabatake, A.; Nakazawa, K.; Kimura, A.; Hiraoka, M. A novel SCN5A mutation associated with idiopathic ventricular fibrillation without typical ECG findings of Brugada syndrome. FEBS Lett. 2000, 479, 29-34. [CrossRef]

22. Fatima, A.; Kaifeng, S.; Dittmann, S.; Xu, G.; Gupta, M.K.; Linke, M.; Zechner, U.; Nguemo, F.; Milting, H.; Farr, M.; et al. The disease-specific phenotype in cardiomyocytes derived from induced pluripotent stem cells of two long QT syndrome type 3 patients. PLoS ONE 2013, 8, e83005. [CrossRef] [PubMed]

23. Glazer, A.M.; Wada, Y.; Li, B.; Muhammad, A.; Kalash, O.R.; O’Neill, M.J.; Shields, T.; Hall, L.; Short, L.; Blair, M.A.; et al High-Throughput Reclassification of SCN5A Variants. Am. J. Hum. Genet. 2020, 107, 111-123. [CrossRef]

24. Gui, J.; Wang, T.; Jones, R.P.; Trump, D.; Zimmer, T.; Lei, M. Multiple loss-of-function mechanisms contribute to SCN5A-related familial sick sinus syndrome. PLoS ONE 2010, 5, e10985. [CrossRef]

25. Kapplinger, J.D.; Giudicessi, J.R.; Ye, D.; Tester, D.J.; Callis, T.E.; Valdivia, C.R.; Makielski, J.C.; Wilde, A.A.; Ackerman, M.J Enhanced Classification of Brugada Syndrome-Associated and Long-QT Syndrome-Associated Genetic Variants in the SCN5AEncoded $\mathrm{Na}(\mathrm{v}) 1.5$ Cardiac Sodium Channel. Circ. Cardiovasc. Genet. 2015, 8, 582-595. [CrossRef]

26. Malan, D.; Zhang, M.; Stallmeyer, B.; Muller, J.; Fleischmann, B.K.; Schulze-Bahr, E.; Sasse, P.; Greber, B. Human iPS cell model of type 3 long QT syndrome recapitulates drug-based phenotype correction. Basic Res. Cardiol. 2016, 111, 14. [CrossRef]

27. Medeiros-Domingo, A.; Tan, B.H.; Iturralde-Torres, P.; Tester, D.J.; Tusie-Luna, T.; Makielski, J.C.; Ackerman, M.J. Unique mixed phenotype and unexpected functional effect revealed by novel compound heterozygosity mutations involving SCN5A. Heart Rhythm 2009, 6, 1170-1175. [CrossRef]

28. Nakajima, T.; Kaneko, Y.; Saito, A.; Ota, M.; Iijima, T.; Kurabayashi, M. Enhanced fast-inactivated state stability of cardiac sodium channels by a novel voltage sensor SCN5A mutation, R1632C, as a cause of atypical Brugada syndrome. Heart Rhythm 2015, 12, 2296-2304. [CrossRef]

29. Tan, B.H.; Iturralde-Torres, P.; Medeiros-Domingo, A.; Nava, S.; Tester, D.J.; Valdivia, C.R.; Tusie-Luna, T.; Ackerman, M.J.; Makielski, J.C. A novel C-terminal truncation SCN5A mutation from a patient with sick sinus syndrome, conduction disorder and ventricular tachycardia. Cardiovasc. Res. 2007, 76, 409-417. [CrossRef]

30. Ahmad, F.; McNally, E.M.; Ackerman, M.J.; Baty, L.C.; Day, S.M.; Kullo, I.J.; Madueme, P.C.; Maron, M.S.; Martinez, M.W.; Salberg, L.; et al. Establishment of Specialized Clinical Cardiovascular Genetics Programs: Recognizing the Need and Meeting Standards: A Scientific Statement from the American Heart Association. Circ. Genom. Precis. Med. 2019, 12, e000054. [CrossRef]

31. Kapplinger, J.D.; Tester, D.J.; Alders, M.; Benito, B.; Berthet, M.; Brugada, J.; Brugada, P.; Fressart, V.; Guerchicoff, A.; Harris-Kerr, C.; et al. An international compendium of mutations in the SCN5A-encoded cardiac sodium channel in patients referred for Brugada syndrome genetic testing. Heart Rhythm 2010, 7, 33-46. [CrossRef] [PubMed]

32. Abe, K.; Machida, T.; Sumitomo, N.; Yamamoto, H.; Ohkubo, K.; Watanabe, I.; Makiyama, T.; Fukae, S.; Kohno, M.; Harrell, D.T.; et al. Sodium channelopathy underlying familial sick sinus syndrome with early onset and predominantly male characteristics. Circ. Arrhythm. Electrophysiol. 2014, 7, 511-517. [CrossRef]

33. Baskar, S.; Ackerman, M.J.; Clements, D.; Mayuga, K.A.; Aziz, P.F. Compound heterozygous mutations in the SCN5A-encoded Nav1.5 cardiac sodium channel resulting in atrial standstill and His-Purkinje system disease. J. Pediatr. 2014, 165, 1050-1052. [CrossRef]

34. Bezzina, C.R.; Rook, M.B.; Groenewegen, W.A.; Herfst, L.J.; van der Wal, A.C.; Lam, J.; Jongsma, H.J.; Wilde, A.A.; Mannens, M.M. Compound heterozygosity for mutations (W156X and R225W) in SCN5A associated with severe cardiac conduction disturbances and degenerative changes in the conduction system. Circ. Res. 2003, 92, 159-168. [CrossRef]

35. Cordeiro, J.M.; Barajas-Martinez, H.; Hong, K.; Burashnikov, E.; Pfeiffer, R.; Orsino, A.M.; Wu, Y.S.; Hu, D.; Brugada, J.; Brugada, P.; et al. Compound heterozygous mutations P336L and I1660V in the human cardiac sodium channel associated with the Brugada syndrome. Circulation 2006, 114, 2026-2033. [CrossRef] [PubMed]

36. Nunez, L.; Barana, A.; Amoros, I.; de la Fuente, M.G.; Dolz-Gaiton, P.; Gomez, R.; Rodriguez-Garcia, I.; Mosquera, I.; Monserrat, L.; Delpon, E.; et al. p.D1690N Nav1.5 rescues p.G1748D mutation gating defects in a compound heterozygous Brugada syndrome patient. Heart Rhythm 2013, 10, 264-272. [CrossRef] [PubMed]

37. Tan, B.Y.; Yong, R.Y.; Barajas-Martinez, H.; Dumaine, R.; Chew, Y.X.; Wasan, P.S.; Ching, C.K.; Ho, K.L.; Gan, L.S.; Morin, N.; et al. A Brugada syndrome proband with compound heterozygote SCN5A mutations identified from a Chinese family in Singapore. Europace 2016, 18, 897-904. [CrossRef]

38. Thongnak, C.; Limprasert, P.; Tangviriyapaiboon, D.; Silvilairat, S.; Puangpetch, A.; Pasomsub, E.; Sukasem, C.; Chantratita, W. Exome Sequencing Identifies Compound Heterozygous Mutations in SCN5A Associated with Congenital Complete Heart Block in the Thai Population. Dis. Markers 2016, 2016, 3684965. [CrossRef] 
39. Ghovanloo, M.R.; Atallah, J.; Escudero, C.A.; Ruben, P.C. Biophysical Characterization of a Novel SCN5A Mutation Associated with an Atypical Phenotype of Atrial and Ventricular Arrhythmias and Sudden Death. Front. Physiol. 2020, 11, 610436. [CrossRef]

40. Grandi, E.; Pandit, S.V.; Voigt, N.; Workman, A.J.; Dobrev, D.; Jalife, J.; Bers, D.M. Human atrial action potential and Ca ${ }^{2+}$ model: $^{2}$ Sinus rhythm and chronic atrial fibrillation. Circ. Res. 2011, 109, 1055-1066. [CrossRef]

41. O'Hara, T.; Virag, L.; Varro, A.; Rudy, Y. Simulation of the undiseased human cardiac ventricular action potential: Model formulation and experimental validation. PLoS Comput. Biol. 2011, 7, e1002061. [CrossRef]

42. Marangoni, S.; Di Resta, C.; Rocchetti, M.; Barile, L.; Rizzetto, R.; Summa, A.; Severi, S.; Sommariva, E.; Pappone, C.; Ferrari, M.; et al. A Brugada syndrome mutation (p.S216L) and its modulation by p.H558R polymorphism: Standard and dynamic characterization. Cardiovasc. Res. 2011, 91, 606-616. [CrossRef] [PubMed]

43. Viswanathan, P.C.; Benson, D.W.; Balser, J.R. A common SCN5A polymorphism modulates the biophysical effects of an SCN5A mutation. J. Clin. Investig. 2003, 111, 341-346. [CrossRef]

44. Ye, B.; Valdivia, C.R.; Ackerman, M.J.; Makielski, J.C. A common human SCN5A polymorphism modifies expression of an arrhythmia causing mutation. Physiol. Genom. 2003, 12, 187-193. [CrossRef]

45. Nijak, A.; Saenen, J.; Labro, A.J.; Schepers, D.; Loeys, B.L.; Alaerts, M. iPSC-Cardiomyocyte Models of Brugada SyndromeAchievements, Challenges and Future Perspectives. Int. J. Mol. Sci. 2021, 22, 2825. [CrossRef]

46. Watanabe, H.; Yang, T.; Stroud, D.M.; Lowe, J.S.; Harris, L.; Atack, T.C.; Wang, D.W.; Hipkens, S.B.; Leake, B.; Hall, L.; et al. Striking In vivo phenotype of a disease-associated human SCN5A mutation producing minimal changes in vitro. Circulation 2011, 124, 1001-1011. [CrossRef] [PubMed]

47. Kamga, M.V.K.; Reppel, M.; Hescheler, J.; Nguemo, F. Modeling genetic cardiac channelopathies using induced pluripotent stem cells-Status quo from an electrophysiological perspective. Biochem. Pharmacol. 2021, 192, 114746. [CrossRef] [PubMed] 